\title{
The Effects of Limosilactobacillus reuteri LR-99 Supplementation on Body Mass Index, Social Communication, Fine Motor Function, and Gut Microbiome Composition in Individuals with Prader-Willi Syndrome: a Randomized Double-Blinded Placebo-Controlled Trial
}

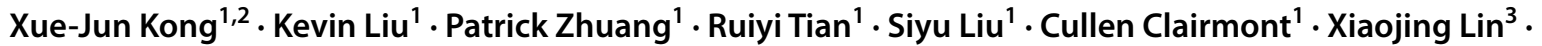

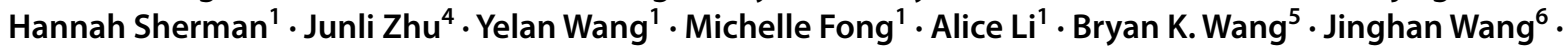 \\ Zhehao $\mathrm{Yu}^{7} \cdot \mathrm{Chen} \mathrm{Shen}^{7} \cdot \mathrm{Xianghua} \mathrm{Cui}^{7} \cdot \mathrm{Hanyu} \mathrm{Cao}^{7} \cdot$ Ting Du$^{7} \cdot \mathrm{Guobin} \mathrm{Wan}^{8} \cdot \mathrm{Xia} \mathrm{Cao}^{7}$
}

Accepted: 12 May 2021 / Published online: 11 June 2021

(c) The Author(s) 2021

\begin{abstract}
Prader-Willi syndrome (PWS) is a rare genetic disorder associated with developmental delay, obesity, and neuropsychiatric comorbidities. Limosilactobacillus reuteri (Lactobacillus reuteri, Lact. reuteri) has demonstrated anti-obesity and antiinflammatory effects in previous studies. In the present study, we aim to evaluate the effects of Lact. reuteri supplementation on body mass index (BMI), social behaviors, and gut microbiota in individuals with PWS. We conducted a 12-week, randomized, double-blind, placebo-controlled trial in 71 individuals with PWS aged 6 to 264 months (64.4 \pm 51.0 months). Participants were randomly assigned to either receive daily Lact. reuteri LR-99 probiotic $\left(6 \times 10^{10}\right.$ colony forming units) or a placebo sachet. Groupwise differences were assessed for BMI, ASQ-3, and GARS-3 at baseline, 6 weeks, and 12 weeks into treatment. Gut microbiome data was analyzed with the QIIME2 software package, and predictive functional profiling was conducted with PICRUSt-2. We found a significant reduction in BMI for the probiotic group at both 6 weeks and 12 weeks relative to the baseline $(P<0.05)$. Furthermore, we observed a significant improvement in social communication and interaction, fine motor function, and total ASQ-3 score in the probiotics group compared to the placebo group $(P<0.05)$. Altered gut microbiota was observed in the probiotic group to favor weight loss and improve gut health. The findings suggest a novel therapeutic potential for Lact. reuteri LR-99 probiotic to modulate BMI, social behaviors, and gut microbiota in Prader-Willi syndrome patients, although further investigation is warranted.

Trial registration Chinese Clinical Trial Registry: ChiCTR1900022646
\end{abstract}

Keywords Prader-Willi syndrome · Body mass index (BMI) - Social communication · Fine motor function · Microbiome Limosilactobacillus reuteri (Lactobacillus reuteri)

\section{Introduction}

Prader-Willi syndrome (PWS) is an uncommon genetic syndrome that affects approximately one out of every 15,000 people [1]. PWS is recognized as the most common genetic

Xue-Jun Kong

xkong1@mgh.harvard.edu

1 Athinoula A. Martinos Center for Biomedical Imaging, Massachusetts General Hospital, Boston, MA, USA

2 Department of Medicine and Psychiatry, Beth Israel Deaconess Medical Center, Boston, MA, USA

3 PWS Care and Support Center, Hangzhou, China cause of life-threatening childhood obesity and often presents with neuropsychiatric symptoms, such as emotional lability, compulsive behavior, and psychosis [1,2]. Morbid obesity and neuropsychiatric complications are leading causes of death or long-term disabilities. Besides some

\footnotetext{
Yale University, New Haven, CT, USA

5 Brandeis University, Waltham, MA, USA

6 New York University, New York, NY, USA

7 The Second Affiliated Hospital of Kunming Medical University, Kunming, Yunnan, China

8 Shenzhen Maternity and Child Healthcare Hospital, Shenzhen, Guangdong, China
} 
reported efficacy of growth hormone [3], the treatments for PWS are mainly behavioral.

The gut microbiota has been implicated in the pathogenesis of obesity and the neuropsychiatric comorbidities in PWS subjects [4]. Although the gut dysbiosis was found to be quite similar in PWS-related obesity and diet-related obesity [5], a recent study identified several bacteria genera that are differentially abundant in the PWS population [6]. Interestingly, when dysbiotic gut microbiota from PWS patients were transplanted into mice, insulin-receptor signaling decreased 2 weeks prior to an increase in body fat composition, indicating that the gut microbiome in PWS may play a role in the development of obesity [7]. Dysbiosis of the gut microbiome has also been associated with neuropsychiatric conditions, such as psychotic and affective disorders [8]. Past research conducted in our laboratory indicated that the microbiome composition even has the potential to serve as a biomarker in the diagnosis and subtyping of ASD [9].

Recent research has shown the potential for probiotics to improve the most debilitating symptomology of PWS. Gut microbiome composition and metabolic disturbance were improved after probiotic administration in overweight adults [10]. However, the effect of probiotics on obesity and in the PWS population remains under debate. An investigation of the probiotic Bifidobacterium lactis in PWS revealed no significant changes in stool frequency or microbiome composition [11]. While studies have found positive effects of Limosilactobacillus reuteri (Lactobacillus reuteri, Lact. reuteri), the probiotic strain administered in this study, on inflammatory and metabolic diseases [12, 13]; the effect of Lact. reuteri is also uncertain, as one study did find a positive correlation between the endogenous abundance of Lact. reuteri and body mass index (BMI) in Mexican children [14]. Notably, a recent study found that Lact. reuteri administration in the PWS population improved fasting insulin concentration and insulin sensitivity and decreased abdominal adiposity in children older than 4.5 years of age [15]. Lactobacillus reuteri has also been shown to exert beneficial effects on the brain and behavior [16]. Previous studies have reported that Lact. reuteri upregulates the neuropeptide hormone oxytocin (OXT), a factor integral to social bonding and reproduction, within a vagus nerve-mediated pathway in mice [17, 18] and humans [19]. Additional research has found that Lact. reuteri acts in a vagus nerve-dependent manner to rescue deficits in social interaction-induced synaptic plasticity via oxytocin signaling modulation in multiple animal models of ASD [20]. Preliminary results reported from the phase III CARE-PWS clinical trial indicated beneficial effects of intranasal oxytocin treatment on hunger, anxiety, and obsessive-compulsive behaviors in patients with PWS; however, this finding has not yet been published. Lactobacillus reuteri probiotic treatment has potential to be another therapeutic option for upregulating oxytocin in patients with PWS and thereby similarly improving symptomology.

In this study, we conducted a randomized, double-blind, placebo-controlled trial to test our hypothesis that probiotics consumption has beneficial effects on obesity, social behaviors, and neurodevelopment in PWS. We enrolled a total of 71 PWS patients to evaluate the efficacy of a Lact. reuteri strain LR-99 on their BMI, psychological measurements, and gut microbiome compositions and functions relative to placebo controls. In addition to potentially supporting a new intervention for patients with PWS, the microbiome composition data collected from this study may shed light on the underlying mechanisms of PWS pathology and the gut-brain axis.

\section{Materials and Methods}

\section{Study Design}

We designed and conducted a randomized, double-blinded, placebo-controlled clinical trial (flowchart, Fig. 1). In this trial, we randomly assigned enrolled PWS participants, with a 1:1 ratio, to either the probiotics or placebo group. We hypothesize that a 12 -week treatment period is sufficient for probiotics supplementation to induce detectable changes. To achieve a statistical power of $80 \%$ for primary outcomes with a large effect size of 0.8 (Cohen's $d$ ) assumed, a total of 52 participants (26 in each arm) were required. We enrolled and randomized 71 subjects (probiotics group $=37$, placebo group $=34$ ), and all subjects with collected data were included in the final intention-treat data analysis.

\section{Ethical Considerations}

The study was conducted in accordance with the Declaration of Helsinki. Ethical approval was issued by the Internal Review Board (IRB) of the Second Affiliated Hospital of Kunming Medical University, Kunming, China (ReviewYJ-2016-06). The present clinical trial of probiotics was registered at the Chinese Clinical Trial Registry (ChiCTR) with registration number ChiCTR1900022646, in which two interventions of different probiotic strains were independently evaluated in the enrolled subjects. The evaluation of effects of the alternative probiotic strain Bifidobacterium Animalis subsp. lactis $B B-11$, which was co-registered with the present study, was previously published [21]. Signed informed consent was obtained from the parents or legal guardians of all the subjects according to the IRB requirements. 


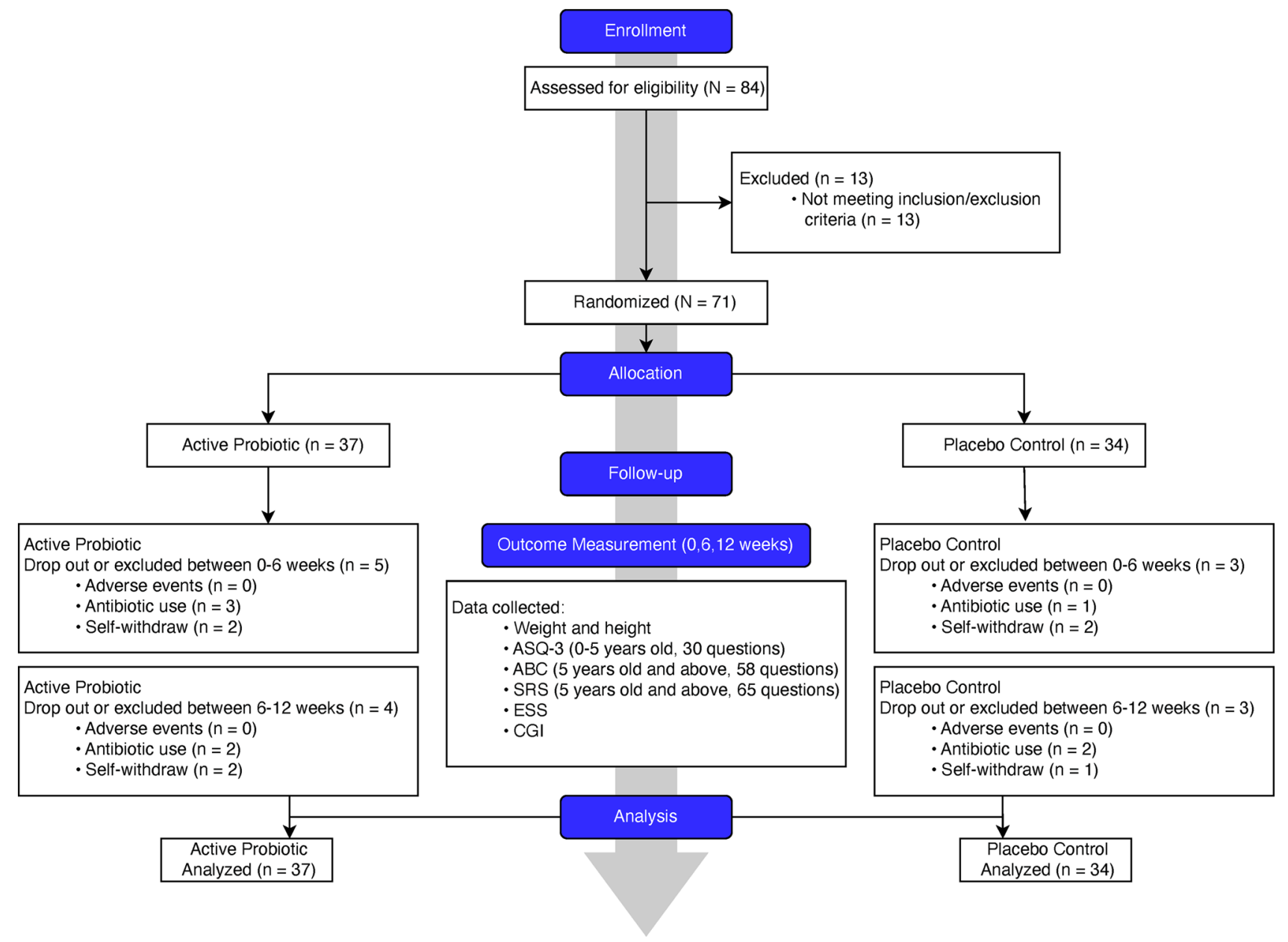

Fig. 1 Flowchart summary of study conduct and participant enrollment and dropout

\section{Participants}

Study participants were recruited through the PWS Care $\&$ Support Centre, located in Zhejiang, China. Participants were included if they met the following criteria: they had been genetically confirmed to have PWS, had not been administered any forms of probiotics for at least 4 weeks, had stable medications for at least 4 weeks, had no planned changes in medications or psychosocial interventions during the trial, had a willingness to provide stool samples in a timely manner, and had a willingness to cooperate with interviews and study procedures. Potential participants were excluded if they had other known genetic disorders, or if they were pregnant or breast-feeding before the study.

\section{Randomization and Blinding}

Randomization and allocation concealment were performed by a statistician who was not part of the research team. Randomization sampling numbers were electronically generated for each deidentified subject. Coded probiotics and placebo of identical appearance were prepared by Beijing Huayuan Academy of Biotechnology to ensure allocation concealment. Both the participants and the research staff/investigators who collected and analyzed the outcome data were blinded to treatment status. Blinding was also maintained by making the probiotics package appear identical to the placebo sachet.

\section{Intervention}

The probiotic Limosilactobacillus reuteri LR-99 (Beijing Huayuan Academy of Biotechnology) was used in the study in the format of a sachet. Each sachet of probiotics supplement contained $3 \times 10^{10}$ colony forming units (CFUs). The placebo was maltodextrin in the sachet with similar color, flavor, and taste as the probiotic sachets. Subjects received one sachet twice a day of either probiotics or placebo for a duration of 12 weeks and were instructed to consume the sachet contents orally with water. 


\section{Outcomes}

\section{Primary Outcomes}

Weight and height measurements were obtained by parents using household scales and collected by the research staff. BMI calculated by weight and height was converted to $\mathrm{z}$-score using age growth references provided by WHO (2006) [22].

Psychological questionnaires were administered by trained and qualified study staff that are bilingual in both English and Mandarin Chinese. The administration of such questionnaires was conducted via interview in Mandarin Chinese.

- Ages and Stages Questionnaires, 3rd Edition (ASQ-3) [23]

The ASQ-3 is one of the most widely available development screening tools for young children. The ASQ-3 has five domains: communication, gross motor, fine motor, problem-solving, and personal-social. Total scores were calculated. We interviewed all subjects younger than 5 years old.

- Gilliam Autism Rating Scale Third Edition (GARS-3) [24]

The GARS-3 consists of 56 items describing characteristic behaviors of individuals with autism. The items are grouped into six sub-scales: restrictive, repetitive behaviors (RRB), social interaction (SI), social communication (SC), emotional responses (ER), cognitive style (CS), and maladaptive speech (MS). Total scores and sub-scales were calculated. We interviewed all subjects older than 3 years old.

\section{Secondary Outcomes: Fecal Microbiome}

Sample Handling and Collection Stool samples were collected with DNA/RNA shield fecal collection tubes (Zymo, Cat\#R1101) containing $1 \mathrm{~mL}$ preservation solution and were transported to the laboratory by ice bags and then frozen at $-80{ }^{\circ} \mathrm{C}$. TIANmap stool DNA kit was used to extract DNA (TIANGEN, Cat\#DP328) according to the manufacturer's instructions, and DNA samples were carefully quantified with a NanoDrop Spectrophotometer. $\mathrm{A}_{260} / \mathrm{A}_{280}$ ratios were also measured to confirm high-purity DNA yield. DNA samples were frozen at $-20{ }^{\circ} \mathrm{C}$ until use.

16S rRNA Gene Amplicon Sequencing The 16S rRNA V3-V4 library was constructed by two rounds of PCR with the following primers: 341F:5'TCGTCGGCAGCG TCAGATGTGTATAAGAGACAGCCTACGGGAGGC AGCAGCCTACGGGNBGCASCAG3' and 805R:5'GTC TCGTGGGCTCGGAGATGTGTATAAGAGACAGTG
ACTACNVGGGTATCTAATCC3' via reaction procedure $\left(95{ }^{\circ} \mathrm{C}\right.$ for $2 \mathrm{~min}$, followed by 25 cycles at $95{ }^{\circ} \mathrm{C}$ for $30 \mathrm{~s}$, $55^{\circ} \mathrm{C}$ for $30 \mathrm{~s}$, and $72{ }^{\circ} \mathrm{C}$ for $30 \mathrm{~s}$, and a final extension at $72{ }^{\circ} \mathrm{C}$ for $5 \mathrm{~min}$ ). $\mathrm{PCR}$ products were purified with $1 \times \mathrm{KAPA}$ AMPure beads (KAPA, Cat\#KK8002). Then, products were put through a second PCR reaction procedure $\left(95^{\circ} \mathrm{C}\right.$ for $2 \mathrm{~min}$, followed by 8 cycles at $95{ }^{\circ} \mathrm{C}$ for $30 \mathrm{~s}, 55^{\circ} \mathrm{C}$ for $30 \mathrm{~s}$, and $72{ }^{\circ} \mathrm{C}$ for $30 \mathrm{~s}$, and a final extension at $72{ }^{\circ} \mathrm{C}$ for $5 \mathrm{~min}$ ). $\mathrm{PCR}$ products were purified with $1 \times \mathrm{KAPA}$ AMPure beads and analyzed using a Bioanalyzer DNA Kit, followed by quantification with real-time PCR. DNA libraries were pooled and sequenced on Illumina MiSeq (Illumina; CA) using a $2 \times 250$ bp paired-end protocol with overlapping reads.

\section{Statistical Analysis}

All raw data were recorded and processed in Microsoft Excel 2007 and R. The presentation of data follows the CONSORT recommendations for reporting results of randomized clinical trials (RCTs). Statistical procedures were carried out using $\alpha=0.05$ as the significance level, and missing data was omitted during statistical analysis.

Baseline demographic features were compared between groups using Pearson's $\chi^{2}$-test. We applied the Wilcoxon rank-sum test to explore the intergroup differences in the z-scores of weight, height, total scores, and sub-scores of ASQ-3, GARS-3, ABC, and SRS at baseline, per-subject changes from 0 to 6 weeks, and per-subject changes from 6 to 12 weeks.

Primary outcomes were analyzed using linear mixed effect models (LME) to assess for differences within each primary outcome over the course of the study (0-6 weeks, 6-12 weeks, and 0-12 weeks) for each group via the $L M E R$ package. For all LME analyses, we included time, age, and gender as fixed effects and a random intercept to account for within-subject correlation due to repeated measures over time. In the case of a significant main effect, Bonferronicorrected pairwise comparisons were conducted.

Secondary outcomes were analyzed using similar methods as that of primary outcomes. In addition, linear regression was performed to check for correlations between clinical indices and microbiome compositions.

Receiver operated characteristic (ROC) curves were constructed via the plotROC package for multiple logistic regression models using either select clinical or predictive functional profiling indices.

\section{Microbiome Data Processing and Analysis}

The sequencing reads were filtered using the QIIME2 (v2019.10) based on quality scores [25]. Deblur was used to denoise with default parameters and obtain an abundance 
table of samples by amplicon sequence variants (ASVs) [26].

Alpha diversity metrics were calculated with QIIME2. Bray-Curtis distance was used to characterize microbiome beta diversity. Taxonomies for ASVs were assigned using the sklearn-based taxonomy classifier trained on the sequences at 99\% similarity level from Greengenes v13.8. Significant differences in the relative abundance of microbial phyla, genera, and alpha diversity between placebo and probiotics groups were identified by Kruskal-Wallis tests. A false discovery rate (FDR) based on the Benjamini-Hochberg $(\mathrm{BH})$ adjustment was applied for multiple comparisons.

PICSRUSt-2 was used to infer microbial functional content based on ASVs' abundant tables and then produced Kyoto Encyclopedia of Genes and Genomes (KEGG) orthologs (KO), enzyme classification numbers, and pathway abundance table [27, 28]. The differential analyses were performed on the fold ratios between probiotics and placebo group with a permutation-based nonparametric test, and the top differential features were rendered and plotted with $\mathrm{Cal}$ our [29]. MaAsLin2 was used to explore per-feature correlations between clinical indices and microbial taxa abundances, and results were adjusted for multiple testing using FDR based on the Benjamini-Hochberg method [30].

All raw data from $16 \mathrm{~s}$ rRNA Illumina amplicon sequencing have been deposited in The National Centre for Biotechnology Information (NCBI) Sequence Read Archive (SRA, PRJNA643297).

\section{Results}

\section{Demographic Features of PWS Participants}

A total of 71 subjects aged $64.4 \pm 51.0$ months (ranging from 6 to 264 months) with genetically confirmed diagnosis of Prader-Willi syndrome. Of which, 37 subjects aged
$65.0 \pm 53.8$ months were randomized to receive active probiotic, Lact. reuteri, while 34 subjects aged $64.0 \pm 49.0$ months were randomized to receive placebo. An overview of the subject age distribution is shown in the figure presented in Online Resource 1. Additionally, due to difficulties in data collection, a total of 56 subjects ( $n=28$ in each arm) have available baseline clinical indices. Groupwise comparisons of baseline age, sex, genotype, and other characteristics did not indicate any significant differences $(P>0.05)$. Detailed demographic characteristics of the enrolled participants are summarized in Table 1.

The study recruitment procedure and dropouts at each study timepoint are illustrated as a flowchart in Fig. 1. No serious or severe adverse events were observed. There were no significant differences found between the two groups in any observed adverse events $(P>0.05)$. Of 71 initially enrolled subjects, 15 dropped over the course of the trial. Eight subjects were dropped due to necessary antibiotics use for mild infection occurrence that is not related to the probiotic, which led to termination; seven of those dropped were due to parent- or self-withdrawal; and none of those dropped were due to adverse effects (Fig. 1).

\section{Effects of Probiotics on BMI and Psychological Measurements}

To assess for within-group longitudinal changes in primary outcomes, we applied Bonferroni-corrected pairwise comparisons on linear mixed effects models, using age, gender, and study timepoint as fixed effects and the subject as a random intercept to account for repeated measures over time. The estimated marginal means of BMI at each study visit are detailed in a table (Online Resource 2). Based on such an analysis, we determined that subjects receiving active probiotic display a significant reduction in BMI. Specifically, such significant differences are uniquely observed in the active
Table 1 Demographic features and baseline characteristics of study participants

\begin{tabular}{lllll}
\hline & & $\begin{array}{l}\text { Active probiotic } \\
(N=37)\end{array}$ & $\begin{array}{l}\text { Placebo control } \\
(N=34)\end{array}$ & $P$-value \\
\hline Age $($ months, $n($ mean \pm SD) $)$ & All Subjects & $28(65 \pm 53.8)$ & $28(64 \pm 49.0)$ & 1.00 \\
& $>5$ years & $12(113 \pm 50.2)$ & $11(113 \pm 42.7)$ & 0.81 \\
& $\leq 5$ years & $16(29 \pm 9.8)$ & $17(32 \pm 11.5)$ & 0.52 \\
Sex $(n(\%))$ & Male & $12(43 \%)$ & $18(64 \%)$ & 0.18 \\
Genotype $(n(\%))$ & Female & $16(57 \%)$ & $10(36 \%)$ & \\
& Deletion & $16(57 \%)$ & $15(54 \%)$ & 0.56 \\
Weight $(\mathrm{kg}$, mean \pm SD) & Disomy & $4(14 \%)$ & $2(7 \%)$ & \\
Height $(\mathrm{cm}$, mean $\pm \mathrm{SD})$ & Other/unknown & $8(29 \%)$ & $11(39 \%)$ & 0.71 \\
BMI $($ mean \pm SD) & & $25.8 \pm 15.3$ & $26.2 \pm 21.0$ & 0.66 \\
\hline
\end{tabular}


Table 2 Pairwise comparisons of change in BMI at 6 weeks and 12 weeks compared to baseline based on linear mixed effects models

\begin{tabular}{lllllll}
\hline Intervention & Contrast & Mean difference & Standard error & $\begin{array}{l}\text { Degrees of } \\
\text { freedom }\end{array}$ & t-Ratio & $P$-Value \\
\hline Active probiotic & $12-6 \mathrm{w}$ & -0.312 & 0.362 & 23.153 & -0.863 & 1.000 \\
& $12-0 \mathrm{w}$ & -1.291 & 0.366 & 30.012 & -3.532 & 0.004 \\
& $6-0 \mathrm{w}$ & -0.979 & 0.361 & 23.562 & -2.710 & 0.037 \\
Placebo control & $12-6 \mathrm{w}$ & -3.912 & 1.929 & 17.072 & -2.028 & 0.175 \\
& $12-0 \mathrm{w}$ & -1.459 & 1.782 & 20.078 & -0.819 & 1.000 \\
& $6-0 \mathrm{w}$ & 2.453 & 1.734 & 18.679 & 1.414 & 0.521 \\
\hline
\end{tabular}

probiotic group for BMI between baseline and week 6 , and between baseline and week 12 (Table $2, P<0.05$ ).

Groupwise comparisons of psychological assessment scores were performed at weeks 0,6 , and 12 via the Wilcoxon rank-sum test. A summary of psychological assessment scores, including GARS-3 and ASQ-3, as well as associated statistics for both groups at weeks 0,6 , and 12 are provided in Table 3.

\section{Changes in Microbiome Composition and Function with Probiotics Intervention}

After sequencing, we obtained a total of 3,198,401 raw reads and an average of 49,206.169 reads per sample (ranging from 29,501 to 71,027 reads per sample). Phylum and genus level variations in gut microbiota composition over the intervention course are shown in Fig. 2A for both the probiotics and placebo groups.

Overall, $\alpha$-diversity determined using Shannon, Simpson, ACE, and Chaol indices did not show any significant group differences (Fig. 2B). However, $\beta$-diversity showed a significant separation with probiotics treatment via permutational multivariate ANOVA (PERMANOVA, $\mathrm{F}=1.9018$; $R^{2}=0.022667 ; P<0.05$, Fig. 2C).

To characterize the change in abundance of potentially clinically significant bacteria over the intervention course, we calculated the log twofold changes of detected and identified gut microbiota. The top 9 most prominent fold changes of gut microbiota abundance agglomerated at the genus level are presented in Fig. 2D.

In an attempt to elucidate the changes in the gut microbiome functional profile between those receiving active

Table 3 Summary of psychological measurements, including the ASQ-3 and GARS-3 measures at study timepoints 0-, 6-, and 12-weeks

\begin{tabular}{|c|c|c|c|c|c|c|c|c|c|}
\hline & \multicolumn{3}{|l|}{0 weeks } & \multicolumn{3}{|l|}{6 weeks } & \multicolumn{3}{|l|}{12 weeks } \\
\hline & $\begin{array}{l}\text { Placebo } \\
\text { control }\end{array}$ & Active probiotic & $P$-Value & Placebo control & Active probiotic & $P$-Value & Placebo control & Active probiotic & $P$-Value \\
\hline \multicolumn{10}{|l|}{ GARS-3 } \\
\hline Overall severity & $2.07 \pm 0.46$ & $1.94 \pm 0.68$ & 0.550 & $2.09 \pm 0.54$ & $2.08 \pm 0.86$ & 0.720 & $2.00 \pm 0.47$ & $1.92 \pm 0.76$ & 1.000 \\
\hline $\begin{array}{l}\text { Cognitive style } \\
\text { (CS) }\end{array}$ & $10.4 \pm 4.21$ & $9.63 \pm 4.35$ & 0.762 & $9.36 \pm 5.54$ & $10.3 \pm 4.17$ & 0.647 & $8.55 \pm 4.11$ & $11.1 \pm 5.59$ & 0.120 \\
\hline $\begin{array}{l}\text { Emotional } \\
\text { responses (ER) }\end{array}$ & $13.5 \pm 4.89$ & $13.4 \pm 5.19$ & 0.895 & $14.0 \pm 3.46$ & $14.4 \pm 6.14$ & 0.703 & $14.9 \pm 2.91$ & $14.3 \pm 4.50$ & 0.849 \\
\hline $\begin{array}{l}\text { Maladaptive } \\
\text { Speech (MS) }\end{array}$ & $5.90 \pm 3.42$ & $6.63 \pm 5.62$ & 0.955 & $6.00 \pm 3.97$ & $7.85 \pm 5.47$ & 0.426 & $5.09 \pm 4.13$ & $6.15 \pm 4.86$ & 0.819 \\
\hline $\begin{array}{l}\text { Restrictive/ } \\
\text { repetitive } \\
\text { behaviors (RRB) }\end{array}$ & $19.4 \pm 7.31$ & $15.9 \pm 8.55$ & 0.193 & $17.8 \pm 8.28$ & $20.2 \pm 7.63$ & 0.623 & $18.1 \pm 7.52$ & $18.5 \pm 9.81$ & 0.820 \\
\hline $\begin{array}{l}\text { Social } \\
\text { communication } \\
\text { (SC) }\end{array}$ & $16.6 \pm 6.37$ & $11.7 \pm 7.40$ & 0.047 & $17.8 \pm 5.64$ & $12.7 \pm 4.96$ & 0.088 & $18.8 \pm 5.93$ & $11.5 \pm 6.01$ & 0.007 \\
\hline $\begin{array}{l}\text { Social interaction } \\
\quad \text { (SI) }\end{array}$ & $11.9 \pm 9.20$ & $6.50 \pm 4.52$ & 0.082 & $14.9 \pm 10.4$ & $10.1 \pm 10.0$ & 0.095 & $13.1 \pm 8.55$ & $7.69 \pm 5.57$ & 0.037 \\
\hline \multicolumn{10}{|l|}{ ASQ-3 } \\
\hline Total score & $119 \pm 61.5$ & $131 \pm 66.0$ & 0.593 & $153 \pm 53.4$ & $178 \pm 44.6$ & 0.485 & $123 \pm 33.3$ & $193 \pm 33.8$ & 0.032 \\
\hline Communication & $30.5 \pm 12.6$ & $33.8 \pm 17.1$ & 0.530 & $42.5 \pm 10.8$ & $41.3 \pm 10.6$ & 0.415 & $32.0 \pm 15.2$ & $45.0 \pm 14.1$ & 0.346 \\
\hline Fine motor & $22.5 \pm 17.7$ & $20.6 \pm 9.43$ & 0.892 & $22.5 \pm 15.4$ & $24.4 \pm 17.4$ & 0.686 & $11.0 \pm 8.22$ & $25.0 \pm 15.3$ & 0.027 \\
\hline Gross motor & $15.0 \pm 16.0$ & $26.3 \pm 24.2$ & 0.392 & $28.3 \pm 18.6$ & $35.6 \pm 14.7$ & 1.000 & $24.0 \pm 10.8$ & $39.3 \pm 15.1$ & 0.245 \\
\hline Personal-social & $28.0 \pm 12.5$ & $26.3 \pm 17.9$ & 0.858 & $29.2 \pm 11.6$ & $39.4 \pm 13.2$ & 0.197 & $31.0 \pm 12.4$ & $40.7 \pm 15.1$ & 0.234 \\
\hline Problem solving & $23.0 \pm 12.7$ & $23.8 \pm 17.5$ & 0.964 & $30.8 \pm 15.3$ & $36.9 \pm 14.1$ & 1.000 & $25.0 \pm 12.7$ & $42.9 \pm 8.09$ & 0.051 \\
\hline
\end{tabular}


probiotic and those receiving placebo, we applied predictive functional profiling and performed groupwise comparisons of mean abundance differences for each identified functional pathway. Several functional pathways were determined to be differentially expressed among subjects receiving active probiotic (Table $4, Q<0.1$ ).

Subsequently, using receiver operated characteristic (ROC) curve analysis, we identified the significant clinical parameters (BMI, social communication, social interaction, total ASQ, fine motor) that can be used as biomarkers for treatment response and characterize subjects either receiving probiotics or placebo (Fig. 3A); the fitted logistic regression model is summarized as a table in Online Resource 3. We then identified several key metagenomic functional pathways that can be used to characterize subjects receiving either active probiotics or placebo (Fig. 3B); the fitted logistic regression model is summarized as a table in Online Resource 4. Classification using clinical indices, including ASQ-3 total and fine motor scores and GARS-3 SC and SI scores, resulted in an AUC of $0.9(95 \% \mathrm{CI}=0.7-1)$. Similarly, classification using select functional features of the gut metagenome resulted in an AUC of 0.801 (95\% CI=0.713-0.899).

\section{Correlation Between Gut Microbiota Abundances and Clinical Indices}

Associations between family and genus level microbiota abundances and clinical indices were assessed via MaAsLin2 as univariate linear correlations. Significant correlations at the family and genus levels against clinical indices for measurements at weeks 6 and 12 combined are reported in Table 5. Specifically, BMI is negatively associated with Bifidobacteriaceae $(R=-0.431, Q<0.1)$ and positively associated with Erysipelotrichaceae family level relative abundances $(R=0.261, Q<0.1)$. Standardized (z-score) of BMI is negatively associated with Faecalibacterium $(R=-0.546, Q<0.1)$ and positively associated with $S u b$ doligranulum genus level relative abundances $(R=0.641$, $Q<0.1$ ). Alistipes genus level relative abundance is found to be negatively associated with both GARS-3 ER $(R=-0.644$, $Q<0.1)$ and RRB scores $(R=-0.595, Q<0.1)$.
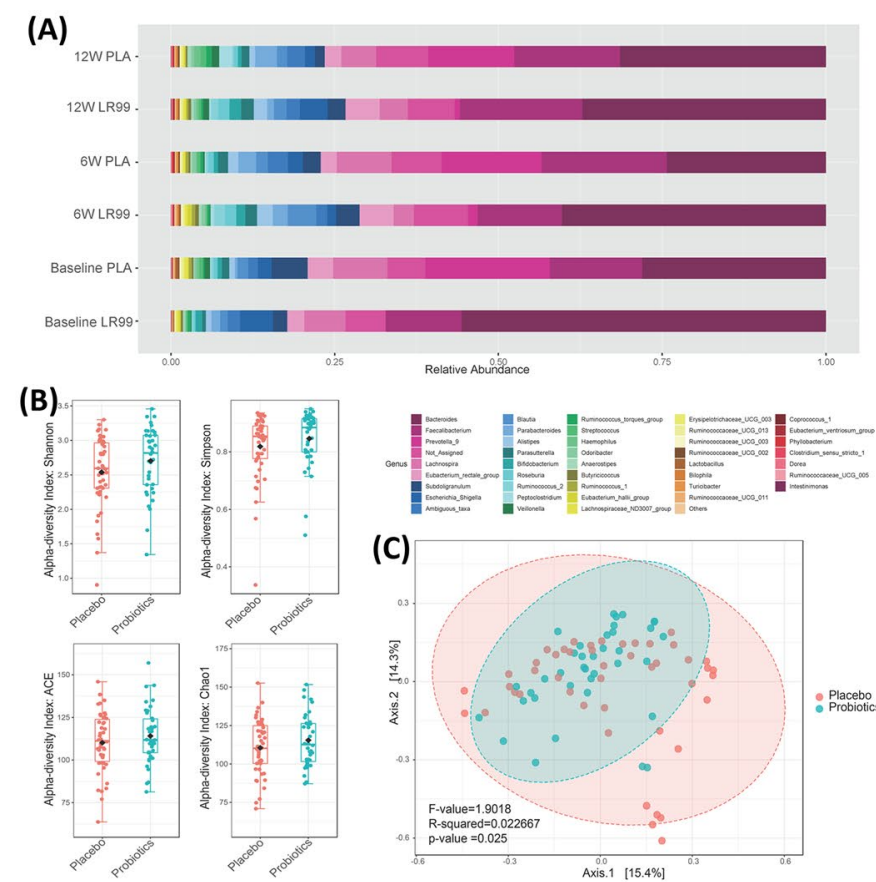

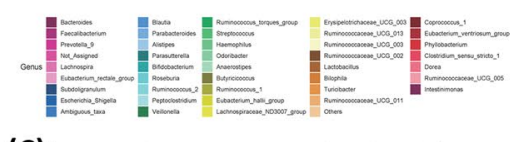

(D)

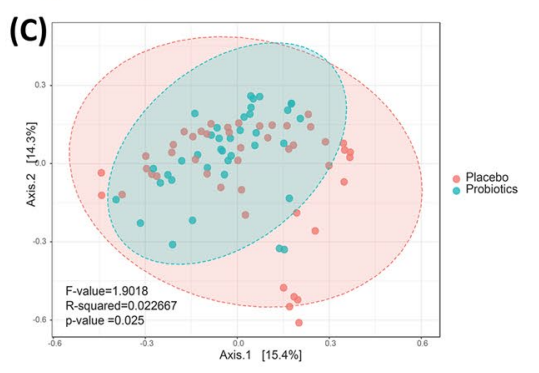

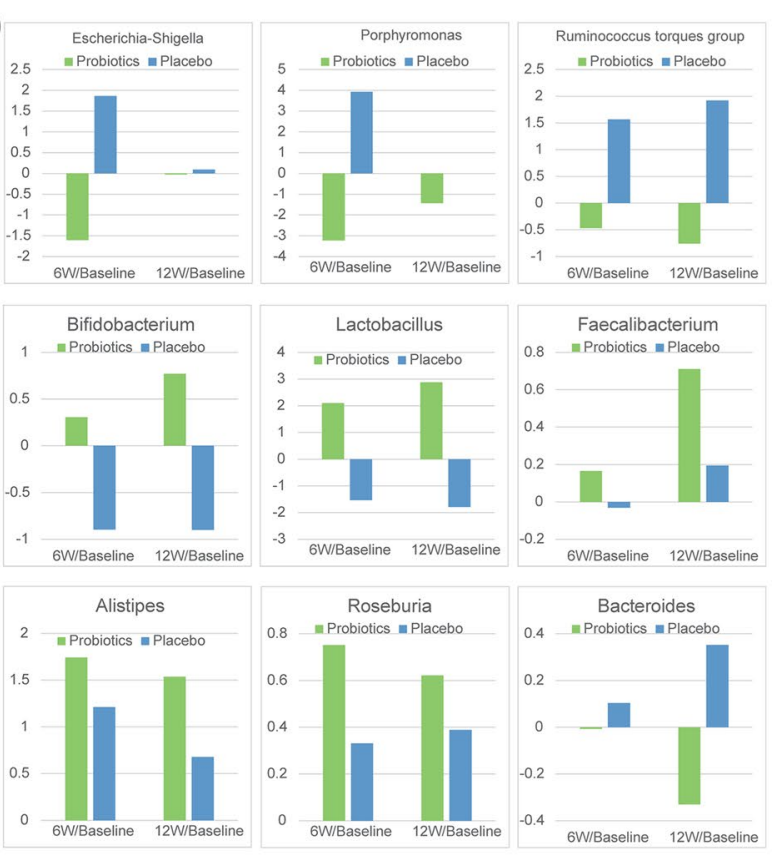

Fig. 2 Overview of genus level relative abundances and measures of microbial diversity. A Relative abundance plots of the gut microbiota at baseline, 6 weeks, and 12 weeks at the genus level. B Mean $\alpha$-diversity measured via Shannon, Simpson, ACE, and Chao1 indices. C $\beta$-diversity with principal coordinates analysis (PCoA) score plots of gut microbial data based on a Bray-Curtis dissimilarity matrix. D Summary of the top 9 most prominent fold changes of relative abundance at genus level over the course of intervention for the probiotics group (green) and placebo (blue). Each bar represents the $\log 2$-transformed relative change of gut microbial abundance of 6 and 12 weeks compared with the baseline 
Table 4 The predicted KEGG enzyme abundance based on PICSRUSt-2 predictive functional profiling for subjects receiving either active probiotic or placebo control. The average abundance of KEGG enzyme abundances is differentially enriched in placebo and probiotics at level 3

\begin{tabular}{|c|c|c|c|c|c|c|}
\hline \multirow[t]{2}{*}{ Feature } & \multicolumn{2}{|l|}{ Mean abundance } & \multirow{2}{*}{$\begin{array}{l}\text { Mean } \\
\text { Difference } \\
(\mathrm{A}-\mathrm{C})\end{array}$} & \multirow[t]{2}{*}{ Mean ratio $(\mathrm{A} / \mathrm{C})$} & \multirow[t]{2}{*}{$P$-Value } & \multirow[t]{2}{*}{$Q$-Value } \\
\hline & Active probiotic & Placebo control & & & & \\
\hline Arachidonic acid metabolism & 218.95 & 360.13 & -141.19 & 0.61 & $<0.001$ & 0.0038 \\
\hline Valine, leucine, and isoleucine biosynthesis & 7469.34 & 6922.96 & 546.37 & 1.08 & 0.0002 & 0.0067 \\
\hline Meiosis (yeast) & 55.66 & 27.30 & 28.36 & 2.04 & 0.0013 & 0.0410 \\
\hline Flavonoid biosynthesis & 49.18 & 20.94 & 28.23 & 2.35 & $<0.001$ & 0.0038 \\
\hline Carotenoid biosynthesis & 71.61 & 20.88 & 50.73 & 3.43 & $<0.001$ & $<0.001$ \\
\hline Steroid biosynthesis & 14.11 & 2.17 & 11.94 & 6.51 & $<0.001$ & 0.0038 \\
\hline Various types of $\mathrm{N}$-glycan biosynthesis & 2.94 & 0.04 & 2.91 & 84.02 & $<0.001$ & $<0.001$ \\
\hline Photosynthesis (antenna proteins) & 90.51 & 0.47 & 90.04 & 192.58 & $<0.001$ & $<0.001$ \\
\hline Calcium signaling pathway & 5.32 & 0.03 & 5.30 & 209.20 & $<0.001$ & $<0.001$ \\
\hline
\end{tabular}

\section{Discussion}

In our 12-week, randomized, double-blind, placebo-controlled trial of 71 subjects with PWS, Lact. reuteri LR-99 significantly reduced the BMI in those receiving the active probiotic compared to those receiving placebo at both 6 weeks $(P<0.05)$ and 12 weeks $(P<0.01)$ of the treatment. One recent study found that Lact. reuteri administration in the PWS population improved insulin sensitivity and decreased abdominal adiposity in children older than 4.5 years of age [15]. However, the mechanisms involved in the induction of such effects remain unclear and may manifest in many different bacteria. The present study provides

(A)

Clinical Indices

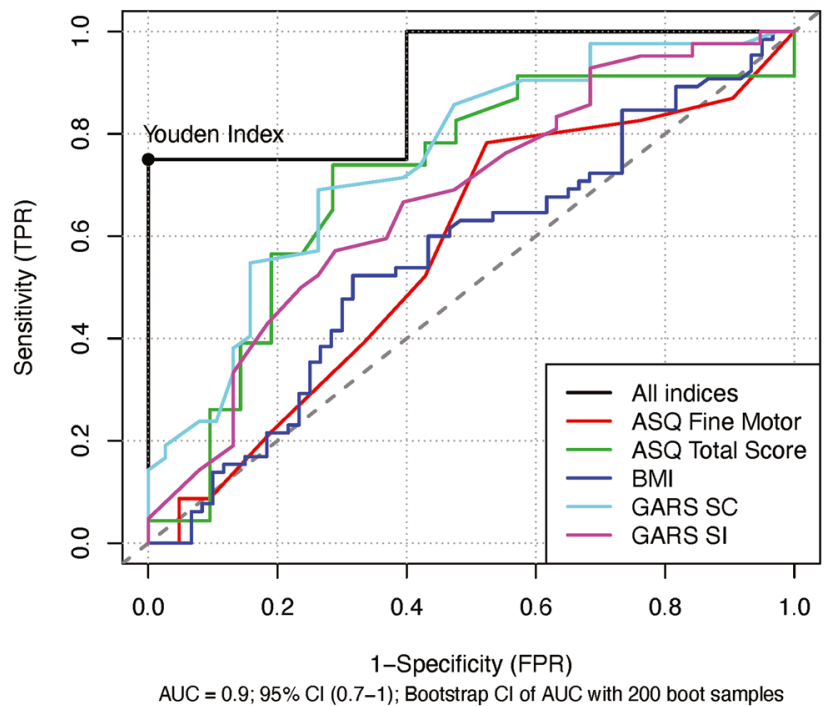

Fig. 3 ROC curve of classification between treatment and placebo groups based on select clinical indices and functional metagenomic features using logistic regression. A Classification using clinical indi- new evidence for Lact. reuteri as a potential, early therapeutic option for PWS that may prevent obesity and related complications. Furthermore, we hope that the study can shed light on the effects and associated mechanistic role of LR-99 in individuals with PWS.

PWS individuals have been found to have absolute or functional growth hormone (GH) deficiency, and GH replacement is currently the most effective treatment for PWS [3]. GH was found not only to increase height, but also decrease body fat and improve cognition, motor, and mental functions [3]. With earlier initiation of GH treatment, increased efficacy and prognostic benefit have been observed [3]. One study has found that probiotics Lact. reuteri could

(B)
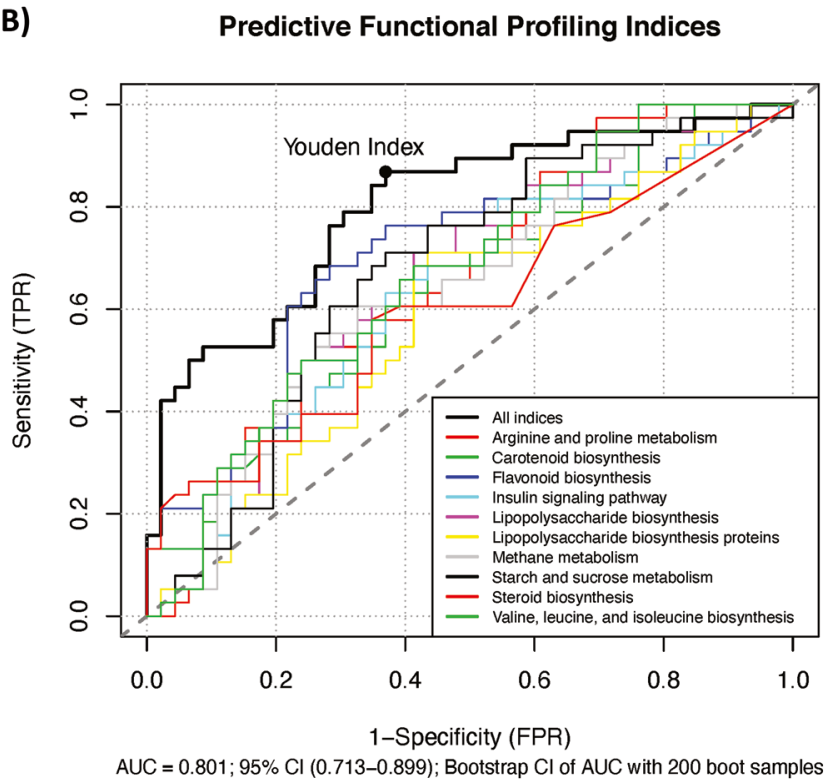

ces, including ASQ-3 total and fine motor scores and GARS-3 SC and SI scores. B Classification using select functional features of the gut metagenome 
Table 5 Summary of significant correlations between genus and family level bacterial abundance and clinical measurements at weeks 6 and 12 combined in the active probiotic group. Taxonomic ranking is

\begin{tabular}{|c|c|c|c|c|c|}
\hline Microbiota & Clinical feature & Coefficient & Standard error & $P$-Value & $Q$-Value \\
\hline Bifidobacteriaceae (f) & BMI & -0.431 & 0.12685 & 0.00433 & 0.06707 \\
\hline Erysipelotrichaceae (f) & BMI & 0.261 & 0.07384 & 0.00329 & 0.06707 \\
\hline Alistipes (g) & ER & -0.644 & 0.11926 & 0.00043 & 0.01217 \\
\hline Alistipes $(\mathrm{g})$ & RRB & -0.595 & 0.14462 & 0.00262 & 0.07335 \\
\hline Faecalibacterium (g) & BMI (z-score) & -0.546 & 0.16112 & 0.00438 & 0.09727 \\
\hline Subdoligranulum (g) & BMI (z-score) & 0.641 & 0.2027 & 0.00695 & 0.09727 \\
\hline
\end{tabular}

labeled in parentheses with "f" denoting family level and "g" denoting genus level microbiota increase growth hormone level in mice [31], which reveals a potential mechanism by which probiotics can improve growth and reduce BMI in PWS patients via promotion of endogenous growth hormone release. Our findings warrant further investigation into the biological mechanisms of probiotics, a promising intervention for PWS with better tolerance and convenience than $\mathrm{GH}$ replacement.

Interestingly, we found that Lact. reuteri intervention significantly improved social communication $(P<0.01)$ and social interaction $(P<0.05)$ compared to controls for those older than 3 years old. Moreover, we found a significant increase in total ASQ-3 score $(P<0.05)$ and fine motor sub-scale $(P<0.05)$ in Lact. reuteri intervention group compared with placebo control group when compared at the last study visit (week 12). While a significant groupwise difference is observed for social communication at baseline (week $0, P<0.05$ ), such differences are observed with a greater magnitude between groups at week $12(P<0.01)$, which is likely a result of Lact. reuteri supplementation. As previously mentioned, Lact. reuteri has been reported to upregulate oxytocin in mice and increase the abundance of OXT-producing cells in the caudal PVN of the hypothalamus in humans $[17,19,20]$. Through vagus nerve-mediated modulation of oxytocin signaling, Lact. reuteri was found to rescue social deficits in multiple mouse models of ASD [20]. Effective treatment of PWS subjects using oxytocin nasal spray in the past [32] and recently in an unpublished phase III CARE-PWS trial provides additional evidence that the improvements we observed in social communication may be related to Lact. reuteri-induced endogenous oxytocin upregulation [32, 33]. These findings of social improvement by Lact. reuteri have not been reported in humans previously, which warrants further study of potential oxytocin signaling deficits involved in the pathogenesis of PWS and other developmental disorders that may also be improved through probiotic supplementation.

The microbiome composition changes we observed with the intervention have been previously linked to weight reduction and inflammatory attenuation. Notably, we found a significant separation of the gut microbiome $\beta$-diversity between the probiotics and the placebo group after treatment. $\beta$-Diversity has been directly correlated with longterm weight loss when adhering to a controlled diet [34]. Significant alteration of microbiome composition in PWS by probiotics has not been reported previously. In fact, administration of the probiotic Bifidobacterium animalis spp. lactis in the PWS population was found to have no significant effect on microbiome composition [11]. Therefore, more research about the variable effects of different probiotic strains on obesity is warranted.

After administration of Lact. reuteri, we also noted a trend of reduction in the abundance of several bacteria including Escherichia-Shigella, Porphyromonas, Ruminococcus torques, and Bacteroides. Escherichia-Shigella is well recognized pathogenic bacteria and is found to be enriched in individuals with obesity and type 2 diabetes [35], in addition to those with autism and co-morbid constipation [36]. The role of periodontal pathogens, including Porphyromonas gingivalis ( $P$. gingivalis), in the onset or exacerbation of systemic diseases, has been demonstrated [37]. Ruminococcus torques is one of the prominent species enriched in people with irritable bowel disease [38]. Bacteroides was found to be enriched in subjects with type 1 diabetes [39]. However, the role of Bacteroides in the pathology of type 2 diabetes and inflammation remains controversial [40, 41].

Conversely, Bifidobacterium, Lactobacillus, Faecalibacterium, Roseburia, and Alistipes each trended towards increased abundance in the gut after Lact. reuteri treatment. Lactobacillus, the genus to which the interventional probiotic belongs, has protective effects against weight gain in humans [42]. Bifidobacterium is widely regarded as beneficial to gut health and weight reduction [43, 44]. Alistipes, although its abundance has been inversely correlated to adiposity, lipid, and glucose homeostasis parameters, [45] has been both negatively and positively associated with to autism spectrum disorders and remains controversial [46, 47]. Roseburia and Faecalibacterium are butyrate-producing, anti-inflammatory bacteria. Roseburia was reported to affect colonic motility, immunity maintenance, and antiinflammatory properties in various metabolic pathways 
and several diseases, including irritable bowel syndrome, obesity, type- 2 diabetes, nervous system conditions, and allergies [48]. Faecalibacterium was found to decrease gut permeability and inflammation [49].

Our findings about the differential abundance of bacterial genera following probiotic treatment interestingly overlapped with the findings from a recent study about the microbiome composition of people with PWS [6]. While they found that Bacteroides was enriched in overweight PWS patients relative to normal weight controls, our study found that Bacteroides was decreased after probiotics treatment. However, their study did find that Escherichia was reduced and Bifidobacterium was enriched in overweight PWS patients, while our study found that Lact. reuteri further reduced and increased the abundance of these probiotics, respectively. These results indicate that different bacterial genera may have a variable contribution to PWS pathogenesis and warrants further investigation.

Furthermore, by using predictive functional gene analysis, we found significant upregulation of calcium signaling, flavonoid biosynthesis, carotenoid biosynthesis, steroid biosynthesis, $\mathrm{N}$-glycan biosynthesis, valine, leucine, and isoleucine biosynthesis with both $P$ - and $Q$-values $<0.05$. Of note, carotenoids, a type of antioxidant, were previously found to have beneficial effects on obesity and obesity-associated pathologies [50]. Further, dietary supplementation with Leu or Ile reduced body weight by regulating lipid metabolismrelated genes and insulin sensitivity and alleviated hepatic steatosis [51].

The insulin signaling pathway and starch and sucrose metabolism were also found to be upregulated with $P<0.05$, but $Q>0.1$. This non-significant trend provides additional evidence in support of the findings by Amat-Bou et al. that Lact. reuteri improves insulin signaling and, in this way, promotes normal weight [15]. However, while the probiotic study conducted by Amat-Bou et al. demonstrated such positive effects in individuals with PWS using the Bifidobacterium strain $\mathrm{BPL} 1$, it is unsurprising that such effects are also observed in the present study of LR-99, as both Bifidobacteria and Lactobacilli have important interconnected functional roles in the gut such as fructose and oligosaccharide metabolism [52]. Further, Peng et al. found that carbohydrate intake, unlike dietary fat or protein, was correlated to changes in microbiome diversity and composition in PWS patients [6]. These results indicate that carbohydrate consumption and metabolism may be keys to the pathogenesis of PWS and the efficacy of probiotic treatment.

The predictive functional gene analysis also showed the significant downregulation of arachidonic acid metabolism with both $P$ and $Q<0.05$. Lipopolysaccharide (LPS) and phosphotransferase system (PTS) were also found to be downregulated with $P<0.05$ but $Q>0.05$. Lipopolysaccharide (LPS), endotoxin from gram-negative pathogenic bacteria such as Escherichia-Shigella, has been reportedly involved in the development of obesity and autism.[53, 54] Taken together, the microbiome composition data and predictive functional gene analysis indicate that the diversity separation caused by Lact. reuteri probiotics treatment favors protection against inflammation, obesity, metabolic syndrome, and ASD.

Using ROC curve analysis, we found the clinical indices, including ASQ-3 total, fine motor scores, GARS-3 SC, and SI scores, resulted in an AUC of $0.9(95 \% \mathrm{CI}=0.7-1)$. Classification using select functional features of the gut metagenome resulted in an AUC of 0.801 (95\% CI $=0.713-0.899)$. The high sensitivity and specificity by which improved clinical indices and changes in gut microbiome composition can distinguish PWS who received Lact. reuteri serves as strong evidence to the efficacy of this probiotic treatment. RRB is one of the core symptoms of ASD and has been reported in as many as $25-40 \%$ of PWS cases [55]. Alistipes was found to be negatively correlated with RRB; a decrease in the relative abundance of Alistipes was found in ASD which is consistent with our finding [46, 54]. Subdoligranulum was found to be positively correlated with BMI, while Faecalibacterium was negatively correlated with BMI. Subdoligranulum was found to be increased in obese mice [56], while individuals with obesity have decreased abundance of Faecalibacterium [57]. Additionally, Bifidobacterium was found to be negatively correlated with BMI, which is expected given its widely recognized effects on promoting gut health and weight reduction [43, 44].

In conclusion, this randomized double-blinded placebo control trial for PWS children showed that treatment with probiotic Lact. reuteri for 12 weeks significantly decreased BMI at week 6 and has more pronounced effects when examined after 12 weeks of administration. Lactobacillus reuteri administration also significantly improved social communication and interaction, fine motor function, and overall development score at week 12 in young children. These novel findings have vital implications for early treatment in PWS. Probiotic treatment also altered microbiome composition and function to favor anti-obesity, anti-inflammation, and influence brain function. The significantly improved clinical indices and functional features of the gut metagenome as a result of probiotic treatment were each found to have predictive value with high specificity and sensitivity.

There are some limitations to the study that deserve consideration. First, despite our adoption of proper recruitment and retention strategies, PWS participant enrolment and retention for this trial were challenging; the sample size was relatively small and limited further subgroup analysis. Second, the broad age range used in this study resulted in high subject population heterogeneity and potentially variable treatment efficacy. Third, assessment of fecal microbiome was not controlled for dietary habits and other relevant 
environmental factors, which may influence the microbial abundances at the individual level. Thus, future studies with larger sample sizes, improved control for environmental factors, and subgroup stratification are warranted. Due to the limitations of the study listed above, further studies are warranted to investigate the mechanism and efficacy of LR-99 probiotic treatment in PWS.

Supplementary Information The online version contains supplementary material available at https://doi.org/10.1007/s12602-021-09800-9.

Acknowledgements We highly appreciate Drs. Gang Fu, Qing Song, Peter Huge, Yuanyuan Liang, and Hui Tan from Baheal Pharmaceutical Group for their generous lab support; we highly appreciate Dr. Heng Yang from Institute of Basic Medical Sciences of Chinese Academy of Medical Sciences and Ms. Yanan Wang from Suzhou Func Biotech Inc. for their great helps of 16S rRNA Gene Amplicon Sequencing; we highly appreciate Dr. Yunfeng Duan from Institute of Microbiology of Chinese Academy of Medical Science for his generous helps in blinded microbiome analysis; and we also appreciate Ms.Ye Zhang from Hebei Xiongnan Biotechnology Co., Ltd. for her help on probiotics and placebo manufacturing and transportation as well as providing the probiotic and placebo products as a free gift.

Author Contribution Conceptualization, Xue-Jun Kong. Data curation, Kevin Liu and Ruiyi Tian. Formal analysis, Kevin Liu and Patrick Zhuang. Funding acquisition, Xue-Jun Kong. Investigation, Xue-Jun Kong, Ruiyi Tian, Siyu Liu, Xiaojing Lin, Junli Zhu, Yelan Wang, Michelle Fong, Jinghan Wang, Zhehao Yu, Chen Shen, Xianghua Cui, Hanyu. Cao, Ting Du, Guobin Wan, and Xia Cao. Methodology, Xue-Jun Kong. Project administration, Xue-Jun Kong and Siyu Liu. Resources, Xue-Jun Kong. Software, Kevin Liu and Patrick Zhuang. Supervision, Xue-Jun Kong. Validation, Kevin Liu and Patrick Zhuang. Visualization, Kevin Liu and Patrick Zhuang. Writingoriginal draft, Xue-Jun Kong. Writing-review and editing, Xue-Jun Kong, Kevin Liu, Cullen Clairmont, Hannah Sherman, Alice Li, and Bryan Wang.

Funding This work was supported by the Massachusetts General Hospital under funding \#233263.

Data Availability All 16 s rRNA Illumina amplicon sequencing data presented in this study are openly available in The National Centre for Biotechnology Information (NCBI) Sequence Read Archive (SRA) with accession number PRJNA643297.

Code Availability Available upon request.

\section{Declarations}

Ethical Approval The study was conducted according to the guidelines of the Declaration of Helsinki and approved by the Institutional Review Board of the Second Affiliated Hospital of Kunming Medical University (Review-YJ-2016-06, February 21, 2019).

Consent to Participate Informed consent was obtained from all subjects involved in the study.

Conflict of Interest The authors declare no competing interests.
Open Access This article is licensed under a Creative Commons Attribution 4.0 International License, which permits use, sharing, adaptation, distribution and reproduction in any medium or format, as long as you give appropriate credit to the original author(s) and the source, provide a link to the Creative Commons licence, and indicate if changes were made. The images or other third party material in this article are included in the article's Creative Commons licence, unless indicated otherwise in a credit line to the material. If material is not included in the article's Creative Commons licence and your intended use is not permitted by statutory regulation or exceeds the permitted use, you will need to obtain permission directly from the copyright holder. To view a copy of this licence, visit http://creativecommons.org/licenses/by/4.0/.

\section{References}

1. Irizarry KA, Miller M, Freemark M, Haqq AM (2016) Prader Willi syndrome genetics, metabolomics, hormonal function, and new approaches to therapy. Adv Pediatr 63:47-77. https://doi.org/ 10.1016/j.yapd.2016.04.005

2. Butler MG, Miller JL, Forster JL (2019) Prader-Willi syndrome clinical genetics, diagnosis and treatment approaches: an update. Curr Pediatric Rev 15:207-244. https://doi.org/10.2174/ 1573396315666190716120925

3. Bakker NE, Lindberg A, Heissler J et al (2017) Growth hormone treatment in children with Prader-Willi syndrome: three years of longitudinal data in prepubertal children and adult height data from the KIGS database. J Clin Endocrinol Metabolism 102:1702-1711. https://doi.org/10.1210/jc.2016-2962

4. Olsson LM, Poitou C, Tremaroli V et al (2020) Gut microbiota of obese subjects with Prader-Willi syndrome is linked to metabolic health. Gut 69:1229-1238. https://doi.org/10.1136/ gutjnl-2019-319322

5. Zhang C, Yin A, Li H et al (2015) Dietary modulation of gut microbiota contributes to alleviation of both genetic and simple obesity in children. EBioMedicine 2:968-984. https://doi.org/10. 1016/j.ebiom.2015.07.007

6. Peng Y, Tan Q, Afhami S et al (2020) The gut microbiota profile in children with Prader-Willi syndrome. Genes-Basel 11:904. https://doi.org/10.3390/genes11080904

7. Deng L, Wang R, Li H et al (2019) miRNA-Gene regulatory network in gnotobiotic mice stimulated by dysbiotic gut microbiota transplanted from a genetically obese child. Front Microbiol 10:1517. https://doi.org/10.3389/fmicb.2019.01517

8. Vindegaard N, Speyer H, Nordentoft M et al (2020) Gut microbial changes of patients with psychotic and affective disorders: a systematic review. Schizophr Res. https://doi.org/10.1016/j.schres. 2019.12.014

9. Kong X, Liu J, Cetinbas M et al (2019) New and preliminary evidence on altered oral and gut microbiota in individuals with autism spectrum disorder (ASD): implications for ASD diagnosis and subtyping based on microbial biomarkers. Nutrients 11:2128. https://doi.org/10.3390/nu11092128

10. Hibberd AA, Yde CC, Ziegler ML et al (2019) Probiotic or synbiotic alters the gut microbiota and metabolism in a randomised controlled trial of weight management in overweight adults. Benef Microbes 10:121-135. https://doi.org/10.3920/bm2018.0028

11. Alyousif Z, Miller JL, Auger J et al (2020) Microbiota profile and efficacy of probiotic supplementation on laxation in adults affected by Prader-Willi syndrome: a randomized, double-blind, crossover trial. Mol Genetics Genom Medicine 8:e1535. https:// doi.org/10.1002/mgg3.1535

12. Tenorio-Jiménez C, Martínez-Ramírez MJ, Castillo-Codes ID et al (2019) Lactobacillus reuteri V3401 reduces inflammatory 
biomarkers and modifies the gastrointestinal microbiome in adults with metabolic syndrome: the PROSIR study. Nutrients 11:1761. https://doi.org/10.3390/nu11081761

13. West CL, Stanisz AM, Mao Y-K et al (2020) Microvesicles from Lactobacillus reuteri (DSM-17938) completely reproduce modulation of gut motility by bacteria in mice. PLoS One 15:e0225481. https://doi.org/10.1371/journal.pone.0225481

14. Huerta-Ávila EE, Ramírez-Silva I, Torres-Sánchez LE et al (2019) High relative abundance of Lactobacillus reuteri and fructose intake are associated with adiposity and cardiometabolic risk factors in children from Mexico City. Nutrients 11:1207. https:// doi.org/10.3390/nu11061207

15. Amat-Bou M, Garcia-Ribera S, Climent E et al (2020) Effects of Bifidobacterium animalis Subsp. lactis (BPL1) supplementation in children and adolescents with Prader-Willi syndrome: A randomized crossover trial. Nutrients 12:3123. https://doi.org/10. 3390/nu12103123

16. Dickerson F, Adamos M, Katsafanas E et al (2018) Adjunctive probiotic microorganisms to prevent rehospitalization in patients with acute mania: a randomized controlled trial. Bipolar Disord 20:614-621. https://doi.org/10.1111/bdi.12652

17. Poutahidis T, Kearney SM, Levkovich T et al (2013) Microbial symbionts accelerate wound healing via the neuropeptide hormone oxytocin. PLoS One 8:e78898. https://doi.org/10.1371/ journal.pone.0078898

18. Poutahidis T, Kleinewietfeld M, Smillie C et al (2013) Microbial reprogramming inhibits Western diet-associated obesity. PLoS One 8:e68596. https://doi.org/10.1371/journal.pone.0068596

19. Varian BJ, Poutahidis T, DiBenedictis BT et al (2017) Microbial lysate upregulates host oxytocin. Brain Behav Immun 61:36-49. https://doi.org/10.1016/j.bbi.2016.11.002

20. Sgritta M, Dooling SW, Buffington SA et al (2018) Mechanisms underlying microbial-mediated changes in social behavior in mouse models of autism spectrum disorder. Neuron 101:246-259. e6. https://doi.org/10.1016/j.neuron.2018.11.018

21. Kong X-J, Wan G, Tian R et al (2021) The effects of probiotic supplementation on anthropometric growth and gut microbiota composition in patients with Prader-Willi syndrome: a randomized double-blinded placebo-controlled trial. Front Nutri 8:587974. https://doi.org/10.3389/fnut.2021.587974

22. GROUP WMGRS, Onis M, (2006) WHO child growth standards based on length/height, weight and age. Acta Paediatr 95:76-85. https://doi.org/10.1111/j.1651-2227.2006.tb02378.x

23. Squires J, Twombley E, Bricker D, Potter L et al (2009) Ages and stages questionnaires (ASQ): A parent-completed child monitoring system (3rd ed.). Baltimore, MD: Brookes

24. Gilliam JE (2014) Gilliam Autism Rating Scale-Third Edition. Austin, TX: PRO-ED

25. Bolyen E, Rideout JR, Dillon MR et al (2019) Reproducible, interactive, scalable and extensible microbiome data science using QIIME 2. Nat Biotechnol 37:852-857. https://doi.org/10.1038/ s41587-019-0209-9

26. Amir A, McDonald D, Navas-Molina JA et al (2017) Deblur rapidly resolves single-nucleotide community sequence patterns. Msystems 2:e00191-e216. https://doi.org/10.1128/msystems. 00191-16

27. Douglas GM, Maffei VJ, Zaneveld J et al (2020) PICRUSt2: an improved and customizable approach for metagenome inference. Biorxiv 672295. https://doi.org/10.1101/672295

28. Kanehisa M, Sato Y, Kawashima M et al (2016) KEGG as a reference resource for gene and protein annotation. Nucleic Acids Res 44:D457-D462. https://doi.org/10.1093/nar/gkv1070

29. Xu ZZ, Amir A, Sanders J et al (2019) Calour: an interactive, microbe-centric analysis tool. mSystems 4(1):e00269-18. https:// doi.org/10.1128/mSystems.00269-18
30. Mallick H, Rahnavard A, McIver LJ et al (2021) Multivariable association discovery in population-scale meta-omics studies. Biorxiv 01(20):427420. https://doi.org/10.1101/2021.01.20. 427420

31. Varian BJ, Goureshetti S, Poutahidis T et al (2016) Beneficial bacteria inhibit cachexia. Oncotarget 7:11803-11816. https://doi. org/10.18632/oncotarget.7730

32. Miller JL, Tamura R, Butler MG et al (2017) Oxytocin treatment in children with Prader-Willi syndrome: a double-blind, placebocontrolled, crossover study. Am J Med Genet A 173:1243-1250. https://doi.org/10.1002/ajmg.a.38160

33. Einfeld SL, Smith E, McGregor IS et al (2014) A double-blind randomized controlled trial of oxytocin nasal spray in Prader Willi syndrome. Am J Med Genet A 164:2232-2239. https://doi.org/10. 1002/ajmg.a.36653

34. Grembi JA, Nguyen LH, Haggerty TD et al (2020) Gut microbiota plasticity is correlated with sustained weight loss on a low-carb or low-fat dietary intervention. Sci Rep 10:1405. https://doi.org/ 10.1038/s41598-020-58000-y

35. Anhê FF, Jensen BAH, Varin TV et al (2020) Type 2 diabetes influences bacterial tissue compartmentalisation in human obesity. Nat Metabolism 2:233-242. https://doi.org/10.1038/ s42255-020-0178-9

36. Eshraghi RS, Deth RC, Mittal R et al (2018) Early disruption of the microbiome leading to decreased antioxidant capacity and epigenetic changes: Implications for the rise in autism. Front Cell Neurosci 12:256. https://doi.org/10.3389/fncel.2018.00256

37. Mulhall H, Huck O, Amar S (2020) Porphyromonas gingivalis, a long-range pathogen: systemic impact and therapeutic implications. Microorganisms 8:869. https://doi.org/10.3390/ microorganisms 8060869

38. Lloyd-Price J, Arze C, Ananthakrishnan AN et al (2019) Multi-omics of the gut microbial ecosystem in inflammatory bowel diseases. Nature 569:655-662. https://doi.org/10.1038/ s41586-019-1237-9

39. Alkanani AK, Hara N, Gottlieb PA et al (2015) Alterations in intestinal microbiota correlate with susceptibility to type 1 diabetes. Diabetes 64:3510-3520. https://doi.org/10.2337/db14-1847

40. Gurung M, Li Z, You H et al (2020) Role of gut microbiota in type 2 diabetes pathophysiology. EBioMedicine 51:102590. https://doi. org/10.1016/j.ebiom.2019.11.051

41. Hiippala K, Kainulainen V, Suutarinen M et al (2020) Isolation of anti-inflammatory and epithelium reinforcing Bacteroides and Parabacteroides Spp. from a healthy fecal donor. Nutrients 12:935. https://doi.org/10.3390/nu12040935

42. Rosing JA, Walker KC, Jensen BAH, Heitmann BL (2017) Oral Lactobacillus counts predict weight gain susceptibility: a 6-year follow-up study. Obes Facts 10:473-482. https://doi.org/10.1159/ 000478095

43. Pedret A, Valls RM, Calderón-Pérez L et al (2019) Effects of daily consumption of the probiotic Bifidobacterium animalis subsp. lactis CECT 8145 on anthropometric adiposity biomarkers in abdominally obese subjects: a randomized controlled trial. Int J Obesity 43:1863-1868. https://doi.org/10.1038/s41366-018-0220-0

44. Uusitupa H-M, Rasinkangas P, Lehtinen MJ et al (2020) Bifidobacterium animalis subsp. lactis 420 for metabolic health: Review of the research. Nutrients 12:892.https://doi.org/10.3390/ nu12040892

45. Garcia-Ribera S, Amat-Bou M, Climent E et al (2020) Specific dietary components and gut microbiota composition are associated with obesity in children and adolescents with PraderWilli syndrome. Nutrients 12:1063. https://doi.org/10.3390/ nu12041063 
46. Strati F, Cavalieri D, Albanese D et al (2017) New evidences on the altered gut microbiota in autism spectrum disorders. Microbiome 5:24. https://doi.org/10.1186/s40168-017-0242-1

47. Angelis MD, Piccolo M, Vannini L et al (2013) Fecal microbiota and metabolome of children with autism and pervasive developmental disorder not otherwise specified. PLoS One 8:e76993. https://doi.org/10.1371/journal.pone.0076993

48. Tamanai-Shacoori Z, Smida I, Bousarghin L et al (2017) Roseburia spp.: a marker of health? Future Microbiol 12:157-170. https://doi.org/10.2217/fmb-2016-0130

49. Mörkl S, Lackner S, Meinitzer A et al (2018) Gut microbiota, dietary intakes and intestinal permeability reflected by serum zonulin in women. Eur J Nutr 57:2985-2997. https://doi.org/10. 1007/s00394-018-1784-0

50. Mounien L, Tourniaire F, Landrier J-F (2019) Anti-obesity effect of carotenoids: Direct impact on adipose tissue and adipose tissuedriven indirect effects. Nutrients 11:1562. https://doi.org/10.3390/ nu11071562

51. Ma Q, Zhou X, Hu L et al (2020) Leucine and isoleucine have similar effects on reducing lipid accumulation, improving insulin sensitivity and increasing the browning of WAT in high-fat dietinduced obese mice. Food Funct 11:2279-2290. https://doi.org/ $10.1039 / \mathrm{c} 9$ fo03084k

52. Gänzle MG, Follador R (2012) Metabolism of oligosaccharides and starch in lactobacilli: a review. Front Microbiol 3:340. https:// doi.org/10.3389/fmicb.2012.00340
53. Hersoug L-G, Møller P, Loft S (2018) Role of microbiota-derived lipopolysaccharide in adipose tissue inflammation, adipocyte size and pyroptosis during obesity. Nutr Res Rev 31:153-163. https:// doi.org/10.1017/s0954422417000269

54. Srikantha P, Mohajeri MH (2019) The possible role of the microbiota-gut-brain-axis in autism spectrum disorder. Int J Mol Sci 20:2115. https://doi.org/10.3390/ijms20092115

55. Bennett JA, Germani T, Haqq AM, Zwaigenbaum L (2015) Autism spectrum disorder in Prader-Willi syndrome: a systematic review. Am J Med Genet A 167:2936-2944. https://doi.org/ 10.1002/ajmg.a.37286

56. Elmassry MM, Chung E, Cao JJ et al (2020) Osteoprotective effect of green tea polyphenols and annatto-extracted tocotrienol in obese mice is associated with enhanced microbiome vitamin K2 biosynthetic pathways. J Nutritional Biochem 86:108492. https:// doi.org/10.1016/j.jnutbio.2020.108492

57. Crovesy L, Masterson D, Rosado EL (2020) Profile of the gut microbiota of adults with obesity: a systematic review. Eur J Clin Nutr 74:1251-1262. https://doi.org/10.1038/s41430-020-0607-6

Publisher's Note Springer Nature remains neutral with regard to jurisdictional claims in published maps and institutional affiliations. 